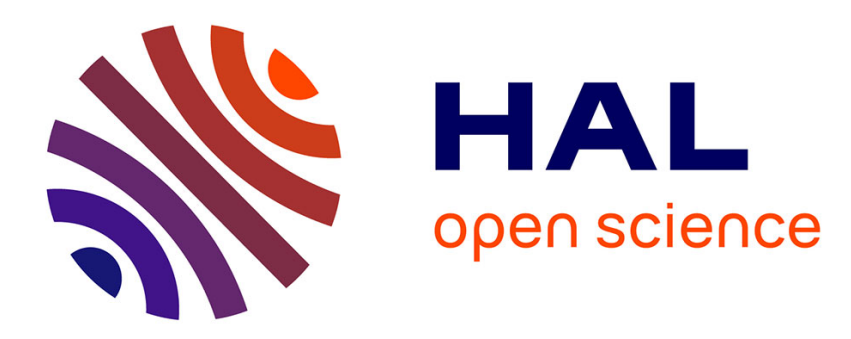

\title{
JÉSUS APRÈS ARTE. RETOUR SUR UNE TRILOGIE
}

Pierre Lassave

\section{To cite this version:}

Pierre Lassave. JÉSUS APRÈS ARTE. RETOUR SUR UNE TRILOGIE. Revue Esprit, 2009, pp.6985. halshs- 03260306

\section{HAL Id: halshs-03260306 https://shs.hal.science/halshs-03260306}

Submitted on 18 Jun 2021

HAL is a multi-disciplinary open access archive for the deposit and dissemination of scientific research documents, whether they are published or not. The documents may come from teaching and research institutions in France or abroad, or from public or private research centers.
L'archive ouverte pluridisciplinaire HAL, est destinée au dépôt et à la diffusion de documents scientifiques de niveau recherche, publiés ou non, émanant des établissements d'enseignement et de recherche français ou étrangers, des laboratoires publics ou privés. 


\section{JÉSUS APRÈS ARTE. RETOUR SUR UNE TRILOGIE}

\section{Pierre Lassave}

Éditions Esprit | «sprit »

2009/7 Juillet | pages 69 à 85

ISSN 0014-0759

ISBN 9782909210780

Article disponible en ligne à l'adresse :

https://www.cairn.info/revue-esprit-2009-7-page-69.htm

Distribution électronique Cairn.info pour Éditions Esprit.

(C) Éditions Esprit. Tous droits réservés pour tous pays.

La reproduction ou représentation de cet article, notamment par photocopie, n'est autorisée que dans les limites des conditions générales d'utilisation du site ou, le cas échéant, des conditions générales de la licence souscrite par votre établissement. Toute autre reproduction ou représentation, en tout ou partie, sous quelque forme et de quelque manière que ce soit, est interdite sauf accord préalable et écrit de l'éditeur, en dehors des cas prévus par la législation en vigueur en France. Il est précisé que son stockage dans une base de données est également interdit. 


\title{
Jésus après Arte. Retour sur une trilogie
}

\author{
Pierre Lassave*
}

C' de Gérard Mordillat et Jérôme Prieur sur la chaîne franco-allemande Arte ont fait leur entrée dans le paysage culturel européen. Corpus Christi, enquête sur une énigme nommée Jésus: douze émissions en début de soirée réparties entre les «semaines saintes» de 1997 et de 1998; L'origine du christianisme, poursuite du documentaire sur les premiers écrits chrétiens (notamment les épîtres de Paul et les Actes des Apôtres), même copieux programme pascal en 2004 (étalé sur deux semaines); L'Apocalypse, terme de la série qui, à l'approche de Noël 2008, aborde la christianisation de l'Empire romain. Soit en tout trente-deux émissions de cinquante-deux minutes chacune dont les deux premiers volets ont attiré un public régulier de plus d'un million de téléspectateurs par soirée.

Un tel score d'audience fut au départ un grand soulagement et un encouragement pour le producteur de la chaîne culturelle car les réalisateurs de ces sujets complexes avaient de surcroît opté pour un abord fort austère, loin du reportage exotique ou du clinquant débat de plateau qui fait recette. Chaque émission consiste en effet en un puzzle de conjectures directement exprimées par des spécialistes inconnus du grand public, exégètes et historiens répondant à des questions que l'on devine à peine derrière les reprises conclusives d'une voix off qui mène l'enquête; cadrage intimiste où chaque locuteur parle seul face à la caméra sur fond noir; plans fixes à peine entrecoupés de plages de respiration où l'objectif caresse quelque papyrus ou codex soumis à la question. Bien qu'appartenant en majorité à des facultés de théologie d'Europe et d'Amérique, les divers savants qui défilent dans le studio (une centaine en tout) partagent

\footnotetext{
* Chercheur au Centre d'études interdisciplinaires des faits religieux (EHEss-CNRs).
} 
avec leurs collègues d'institutions laïques un même sens critique; ils prennent ainsi leur matériau principal, les textes canoniques, comme témoins d'une longue histoire forgée par la rencontre de la foi et du pouvoir et qui s'éprouve aujourd'hui dans le conflit des interprétations; sens critique qui va à l'encontre du catéchisme en même temps qu'il combat la perte de mémoire collective.

Comment discerner ce qui sépare Jésus de Nazareth de ce qu’il est devenu sous la figure de Jésus-Christ dans la tradition chrétienne? Son corps crucifié est-il celui d'un dieu, d'un homme ou d'un texte? s’interroge en substance la première série. Qui de Jésus, de Paul, de Pierre ou de Jacques est le fondateur de la nouvelle religion? Celle-ci s'est-elle dûment approprié l'héritage juif? poursuit la deuxième. Comment et pourquoi l'attente imminente de la fin des temps qui anime une petite secte juive a-t-elle pu aboutir en l'espace de trois siècles à la religion officielle de l'Empire romain? anime la troisième. Questionnaire général que les érudits sollicités font rebondir en une cascade d'autres questions pour le plus grand bonheur des téléspectateurs curieux: Jésus voulait-il vraiment renverser le Temple de Jérusalem? Pourquoi Paul, le premier auteur chrétien, n'évoque jamais le passage de la mort à la vie mais uniquement les apparitions du Ressuscité? Qu'est-ce qui sépare les Hébreux des Hellènes dans la première communauté de Jérusalem? Pourquoi, au deuxième siècle, les différents adeptes prétendent-ils incarner le «vrai Israël» au lieu de rompre purement et simplement avec l'héritage judaïque? Pourquoi certaines formes de christianisme comme la «gnose» ont-elles disparu? Comment et pourquoi l'empereur Constantin s'est-il placé sous la protection du dieu chrétien lors de la bataille du Pont Milvius en 312? La liste est innombrable et s'allonge à chaque nouvelle conjecture.

Comment alors à notre tour comprendre qu'un tel questionnaire sans fin qui peut encore heurter la foi des croyants ait pu rencontrer un public inattendu et susciter surtout un accompagnement médiatique hors du commun pour ce type d'émission culturelle aux marges de l'industrie du divertissement? L'examen des volumineuses revues de presse qui suivent les apparitions télévisées est à cet égard instructif $^{l}$. Il montre notamment les raisons de l'événement, ses protagonistes de premier plan (les réalisateurs et les chercheurs) et de second plan (les religieux sur leurs gardes, les journalistes à l'épreuve) avec en toile de fond un public à peine discernable derrière d'abondantes louanges et quelques objections. L'étalement sur une dizaine d'années de ce que l'on a appelé un vrai «spectacle de

1. Soit quatre épais dossiers successifs établis par le service de presse d'Arte (500 à 800 pages chacun, le plus fourni étant celui de 2004 et le moins celui de 2008). Je remercie Jérôme Prieur pour m'avoir aimablement facilité l'accès de ces sources. 
l'intelligence» en quatre grandes plages de rendez-vous nocturnes dégage avec le recul du temps divers traits et inflexions notables.

Après le premier effet de surprise de Corpus Christi qui révèle au grand jour la profondeur d'un savoir méconnu et la curiosité d'un public exigeant, vient le temps des réserves du côté des clercs et des savants hors du champ de la caméra. Mais les circonstances de la sortie de L'origine du christianisme (momentanément opposée côté raison à la violence sanguinolente de la Passion du Christ selon Mel Gibson) assoient la réputation de sérieux de la série. Avec L'Apocalypse qui clôt l'enquête, la trilogie fait de ses deux réalisateurs des experts en christianisme antique en lieu et place de médiateurs défaillants. Mais leur formule fétiche, «Jésus annonçait le Royaume et c'est l'Église qui est venue», qui reprend une expression d'Alfred Loisy rendue célèbre par ses adversaires «antimodernistes» au début du siècle dernier, va hérisser quelques universitaires pour avoir été sortie de son contexte et avoir surtout réduit à deux termes un processus autrement complexe ${ }^{2}$. Voyons tout cela de plus près.

\section{Les réalisateurs, les exégètes et les journalistes}

Un tiers du millier d'articles de presse se compose d'entretiens des deux réalisateurs. L'inévitable effet de répétition qui en résulte, notamment lors du troisième volet, ne sert pas moins l'analyse. Nos «cinéastes écrivains» férus de littérature, qui ont déjà réalisé ensemble un documentaire sur la mémoire d'Antonin Artaud ${ }^{3}$, voulaient d'abord s'engager dans un thriller savant sur le fameux «suaire de Turin » qui, bien que daté du Moyen Âge par le carbone 14, ne laisse pas les consciences et les journaux tranquilles.

C'est en préparant ce projet qu'ils découvrent incidemment la complexité du texte évangélique. Le chanoine érudit Ulysse Chevalier avait ainsi montré au début du siècle dernier qu'il n'était aucunement question de suaire mais de bandelettes enveloppant le cadavre de Jésus et que selon l'usage juif indiqué par le texte (Jean) tout linge devait être brûlé lors de l'ensevelissement. De lectures en découvertes, l'énigme du texte évangélique devient centrale au point de remplacer le suaire. Un texte fait de fragments de mémoire raboutés à plusieurs mains et qui fait de Jésus le juif de Galilée un Christ universel. En ces années 1990 de regain d'intérêt public pour les traditions religieuses, affaire du voile oblige, mesurer l'écart entre les

2. Alfred Loisy (1857-1940), exégète catholique excommunié en 1908 puis devenu professeur au Collège de France. La phrase incriminée figure dans l'Évangile et l'Église (1902), petit livre de réfutation des thèses essentialistes du théologien protestant libéral Adolf von Harnack. Louvrage ne fit pas moins scandale au sein de l'Église catholique pour la critique historique à laquelle il soumettait les textes et les dogmes.

3. La Véritable histoire d'Artaud le Mômo, Arte vidéo, 1994. 
hypothèses de la science historique et les fausses certitudes des lieux communs sur Jésus-Christ vaut plusieurs années de lectures et de dialogues avec les chercheurs.

Les cinéastes enquêteurs en ressortent en mettant en scène une pléiade de savants s'exprimant à voix haute, toiture d'hypothèses emboîtées comme des tuiles, formule documentaire qui fait mouche quand elle réussit à capter l'attention des plus curieux dès lors livrés à se faire leur propre opinion. Rien pourtant ne destinait les réalisateurs «non croyants » à un tel investissement de longue durée qui les fait prendre dans leur entourage pour des grenouilles de bénitier. Excepté le désir d'en découdre avec la légende, de révéler comment le discours religieux a partie liée au politique, de célébrer l'alliance du doute historique et de l'empathie littéraire forgée depuis plus d'un siècle à l'ombre des universités.

Rétrospectivement, si la formule documentaire n'a pas varié depuis le début, les cinéastes admettent avoir appris beaucoup en quinze ans: les contradictions entre textes, hier prises pour drapeau de la critique, se sont transformées au fil du temps en perles qui réfractent la mémoire vivante des disputes passées. Au contact prolongé des exégètes, ils savent désormais que plus on avance dans la connaissance plus le doute s'installe sur sa validité. Ils se demandent pour finir si tout ce travail réalisé sur la mémoire collective n'a pas profité d'une fenêtre de curiosité à la fin du siècle dernier qui semble s'être assez vite refermée au début du siècle actuel où l'on assiste à un net raidissement du rapport public aux religions, chacune se repliant sur ses traditions.

D'emblée, diverses plumes en vue se disent fascinées par la mosaïque de visages qui parlent à l'esprit, plutôt âgés mais pas seulement masculins: "scoliastes inspirés en compétition fraternelle ${ }^{4}$ ", "penchés sur le mystère de caractères grecs ou araméens et qui, une semaine durant, répètent la même chose : ils ne savent pas... Puech, Grelot, Perrot, Nodet, Geoltrain: ces visages dont Corpus Christi ne nous livre que les patronymes ont employé leur vie à se battre avec des signes effacés sur un bout de chiffon... Que ne donnerait-on pour pénétrer leurs silences et leurs rêves ${ }^{5}$ !». L'ascèse de la quête d'une vérité improbable et qui fait violence à sa propre foi fait système avec la sobriété «janséniste» de la mise en scène. La fixité des portraits libère par contraste nos "petits Icare qui s'envolent et surplombent le dédale d'un texte labyrinthique grâce aux ailes de leur érudition, ils nous épatent autant qu'ils se débattent à l'intérieur d'un discours qui les dépasse ${ }^{6} »$.

4. Bernard-Henri Lévy, Le Point, 4 avril 1997.

5. Daniel Schneidermann, Le Monde-Radio télé, 31 mars 1997.

6. Nicolas Truong, Le Monde de l'éducation, avril 1998. 
Historiens, exégètes, théologiens ou archéologues d'origines et d'obédiences multiples (catholiques, protestants, juifs, agnostiques), de localisations variées (universitaires français, allemands, suisses, américains, anglais, israéliens), aux identités distinctes ou mêlées, ces «éclaireurs du signe sortis de l'ombre » vont un temps être invités par les rédactions éblouies. Quelques prêtres savants vont ainsi jusqu'à confesser que la résurrection du Christ ne relève pas à leurs yeux de l'histoire mais de la théologie. Les pairs qui n'ont pas participé à l'émission sont enclins à épingler le parti pris du questionnaire, par exemple la réduction de l'événement spirituel à une lutte entre communautés rivales qui manipulent diversement la mémoire de Jésus.

$\mathrm{Au}$ fil des trois séries, si les virtuoses de l'exégèse crèvent toujours l'écran de leurs doutes éloquents, ils se font plus rares dans la presse, cédant volontiers la place aux réalisateurs qui courent d'un entretien à l'autre pour pallier au déficit journalistique croissant. Sans doute certains d'entre eux ne veulent-ils plus se voir reprocher par leurs pairs d'avoir cédé à la tentation médiatique. D'autres vont jusqu'à évoquer à demi-mot le montage réducteur de leur parole en insistant sur les divergences entre les chercheurs et les réalisateurs. Un montage qui aurait ainsi sous-estimé la "fécondité » du message de Jésus bien au-delà des seules circonstances historiques de son énonciation et de sa transmission, qui aurait également réduit la multiplicité des premiers engagements chrétiens dans le bassin méditerranéen à une lutte abstraite entre orthodoxes et hérétiques.

La presse salue dans un bel ensemble mécanique la qualité rare d'une série qui lève opportunément un coin de voile sur des sources trop longtemps connues des seuls spécialistes. Les plus chauds partisans se recrutent plutôt à l'aile gauche et côté professions intellectuelles (Libération, Télérama, L'Humanité, Le Nouvel Observateur, Inrockuptibles). Les plus fortes réserves proviennent au fil du temps des organes proches de l'Église bien que sous forme de points d'opinion engageant plus ou moins l'institution (Pèlerin, La Vie); le quotidien La Croix lui consacre cependant de copieux dossiers où l'éloge d'un prélat voisine avec la critique sévère d'un autre. L'hostilité des catholiques traditionalistes ne fait pas défaut (Famille chrétienne, L'Homme nouveau), relayée par les feuilles d'extrême droite (Minute) ou partiellement hébergée au Figaro (dont certains journalistes ne cachent pas par ailleurs leur enthousiasme). Au milieu de l'espace d'opinion, les jugements positifs sont à peine atténués soit pour préciser les limites de l'enquête (Le Monde, Le Monde de la Bible, Réforme) soit pour avertir le lecteur non initié de ses difficultés d'accès ( $L e$ Parisien). La presse régionale épouse ces contours en insistant sur la formation culturelle que les séries dispensent à grande échelle rejoignant en cela la thématique principale des journaux allemands, suisses, belges ou québécois. 
L'intitulé de chaque partie de la trilogie polarise la sémantique des titres : l'énigmatique Corpus Christi ravive ainsi le thème de l'enquête policière sur une affaire jamais résolue («L'affaire Jésus rebondit sur Arte», «Deux auteurs en quête de personnage», «La vie de Jésus comme un polar»). L'origine du christianisme traduit ensuite les apôtres au tribunal de l'histoire («Le christianisme en version polémique», "Querelles de famille», «À la recherche du mystère des origines»). L'Apocalypse enfin conjugue la fin des temps avec celle de l'enquête, lorsque l'Église a pris la place du Royaume annoncé («Retour à la fin des temps », "Apocalypse cathodique», "L'empire du soupçon»). La complexité du texte occupe le devant de la première série, la judéité de Jésus nourrit l'attrait polémique de la seconde, la conversion de Constantin fait définitivement basculer l'histoire de Jésus dans des affaires d'empire.

À la différence d'une autre volumineuse revue de presse, voisine de notre affaire (la réception contrastée de la Nouvelle Traduction de la Bible ou «Bible des écrivains» en 2001), la polémique suscitée par les attaques en provenance du bastion traditionaliste n'a pas animé ou rythmé le concert général ${ }^{7}$. Certes, la seconde série agite le drapeau de l'antijudaïsme des évangiles comme fondement de l'antisémitisme historique et l'on voit alors les traditionalistes afficher effrontément leur préférence pour la Passion selon Mel Gibson tandis qu'à l'opposé les porte-parole de la communauté juive saluent l'œuvre de «réparation morale» entreprise par Arte (L'Actualité juive, L'Arche). Mais c'est la richesse et la complexité du dossier qui semblent prévaloir ici, embarrassant les Églises en quête d'un point de vue lisible, et sollicitant les journalistes dépassés à faire appel aux experts. Les rares chroniqueurs spécialisés dans les affaires religieuses s'en sortent en élargissant le sujet à la reconnaissance réciproque entre juifs et chrétiens (Le Monde, Le Monde des religions), se fendant parfois de copieux dossiers de contre-enquête pour satisfaire la curiosité montante du public (L'Express, Le Point). Mais avant de préciser les critiques qui cheminent et se cristallisent au fil du temps, il n'est sans doute pas inutile de revenir sur les cas de figure en jeu, à travers les principaux schémas construits par les séries.

7. Pierre Lassave, «Les tribulations d'une Bible dans les médias", Archives de sciences sociales des religions, $\mathrm{n}^{\circ} 134,2006$, p. 9-35. Cette nouvelle traduction littéraire qui a fait couler beaucoup d'encre dès sa publication chez Bayard en 2001 a trouvé son public en sachant allier des visées apparemment contradictoires: volonté missionnaire, expérimentation littéraire et investissement éditorial. L'abondante revue de presse qui en résulte montre notamment que les reproches virulents de profanation du dépôt sacré émanant du camp conservateur ont paradoxalement rehaussé et élargi la notoriété de l'entreprise. 


\section{Jésus, le Royaume, l'Église}

Les trois essais successivement écrits par nos réalisateurs précisent leur point de vue ${ }^{8}$. Jésus contre Jésus (1999), le plus analytique au plan littéraire, oppose le prophète itinérant de Galilée au sauveur chrétien des nations; Jésus après Jésus (2004), plus historique, transforme l'opposition en succession à travers les querelles de mémoire que se livrent entre eux les disciples du maître disparu; Jésus sans Jésus (2008), plus synthétique, retrace les étapes de l'alliance décisive entre un de ces courants rivaux et le pouvoir impérial. Au terme de la métamorphose, le judéen sans visage mais aux paraboles si frappantes est prêt pour sa longue carrière iconique de jeune thaumaturge barbu au visage rayonnant. Le Royaume qu'il annonce a fait couler beaucoup d'encre. Nos réalisateurs y reviennent dans un chapitre de leur second essai pour relever la double ambivalence de ce symbole central de la prédication: Royaume de plénitude et de justice à venir ou déjà là, Royaume terrestre ou céleste. Les évangélistes oscillent entre la restauration imminente de la royauté davidique, le paradis attendu de la fin des temps ou la nouvelle naissance personnelle qu'induit sa rencontre intime.

Les essayistes font écho à l'exégèse historique en précisant que le Jésus qui parle d'un royaume invisible chez Jean n'est plus le prophète galiléen mais ce fameux «Jésus après Jésus", le Christ qui a surmonté l'échec de la prédication terrestre et son infamante crucifixion, Ressuscité qui habite désormais les espérances de salut. S'employant à leur tour à une nouvelle synthèse de conjectures sans fin, ils reconstituent la logique des visions du temps et de la vérité qui s'affrontent chez les disciples, notamment lorsque les textes cachent plus ou moins les conflits groupusculaires entre l'observance de la loi judaïque et l'universalisation de la foi (controverses d'Antioche puis de Jérusalem sur le fait de savoir si un adepte du Christ Jésus peut ou non s'affranchir de la circoncision). En confrontant étroitement les Actes des apôtres aux épîtres de Paul, ils soulignent l'habileté du premier rédacteur à gommer «sournoisement » les divergences profondes entre Paul, Jacques et Pierre.

De même que Corpus Christi refaisait l'histoire à partir de six versets centraux de la Passion selon Jean (Jn 19,17-22), de même l'Apocalypse finale sert-elle de piste d'envol au-dessus des quatre premiers siècles qui ont fait l'Église. Livre d'images hallucinantes qui reflète un climat violent de résistance contre les puissances du mal, Rome d'un côté, la «Synagogue de Satan » de l'autre (qui serait non pas le

8. Gérard Mordillat, Jérôme Prieur, Jésus contre Jésus. Essai, Paris, Le Seuil, 1999; Jésus après Jésus. L'origine du christianisme, Paris, Le Seuil, 2004; Jésus sans Jésus. La christianisation de l'Empire romain, Paris, Le Seuil-Arte éditions, 2008. 
lieu du peuple déicide mais au contraire celui de la déviation paulinienne qui abandonne la loi mosaïque). C'est en fait à rebours de ce brûlot qui a failli ne pas figurer dans le canon biblique que l'histoire de l'alliance entre évêques chrétiens et empereurs romains se met en marche. Les persécutions et les hérésies l'activent paradoxalement, martyrologes édifiants d'un côté, apologies conquérantes de l'autre.

À grandes enjambées, nos essayistes parcourent les témoignages sanglants nimbés de légende et rendent visite aux multiples communautés qui se réclament différemment d'un Christ, homme et dieu ou dieu aux apparences humaines. Ils mettent ainsi l'accent sur la condamnation de l'hérésie marcionite qui voulait rompre définitivement avec l'héritage judaïque et se disent que si Marcion avait gagné, l'antijudaïsme chrétien aurait fait long feu. La promotion d'une religion de concorde et de justice, loyale à César, par une élite d'apologètes, les «Pères de l'Église », a finalement raison des dissensions de cour. Le jeune Constantin l'adopte pour prendre le pouvoir (le fameux chrisme victorieux au Pont Milvius); Théodose I ${ }^{\text {er }}$ l'érige en 380 comme religion d'empire. À la même époque, le symbole trinitaire et les bases du canon se fondent lors de conciles d'évêques en présence de l'empereur. Mais après le «sac de Rome» (410), l'Église va prendre ses distances avec le pouvoir temporel: avec Augustin l'auctoritas de la communauté des saints tournés vers la cité céleste se détache de la potestas rivée à la cité terrestre.

Tout au long de leur plongée dans les sources chrétiennes, nos écrivains cinéastes ont pimenté leurs essais (et la voix off qui guide l'enquête) de formules qui font mouche dans les médias: Jésus «trompé » par son Église; la «captation de l'héritage» judaïque par la «mante religieuse» chrétienne; la «sainte alliance» de l'Empire et de l'Église qui «a fait plus de bourreaux qu'elle n'a fait de martyrs» (formule empruntée à Henri Guillemin); le christianisme comme «aberration historique» au sens d'un phénomène qui s'est produit contre toute logique. Provocations à l'encontre du respect dû aux convictions religieuses mais qui croient d'abord servir l'intelligence des origines.

\section{Clercs et publics}

Lors du premier Corpus Christi, les médias religieux sollicitent leurs experts, exégètes ou théologiens. Ceux-ci, agréablement surpris par le sérieux de l'émission, délivrent çà et là quelques leçons de lecture à certains fidèles qui s'offusquent de voir leur Jésus «disséqué comme un insecte par de savants entomologistes ». À la seconde partie, l'année suivante (1998), les avis commencent à se formaliser. Récupérant une part du malaise des fidèles face à la mise en doute 
des sources de leur foi, les voix traditionalistes s'insurgent contre le "négationnisme» de l'émission et mettent outrageusement en parallèle les Évangiles et la Shoah. Comme pour prévenir cette confusion délétère, l'Église catholique lance dans ses diocèses des réunionsdébats autour de la série (présentation en avant-première à l'Institut catholique de Paris en 1998). $\mathrm{M}^{\mathrm{gr}}$ Eyt encourage les initiatives: «N'ayons pas peur des Écritures! La foi ne justifie pas la paresse intellectuelle...»

Mais quelques années plus tard, lors de la série suivante qui scrute les Évangiles comme des «écrits de propagande", une note pour la Conférence doctrinale des évêques de France exprime pour la première fois son point de vue (communiqué du 23 mars 2004). La qualité de l'enquête historique est reconnue en même temps que ses limites. Son optique «positiviste» (dégager le Jésus de Nazareth du Christ de l'Église naissante) l'empêche ainsi de prendre la mesure du drame existentiel qui se joue avec la Résurrection et qui engendre une interminable chaîne d'interprétations qui se poursuit encore aujourd'hui sous des formes œecuméniques. Il est à cet égard « inexact» de présenter Paul comme s'opposant à la loi judaïque ou comme celui qui a occulté le message de Jésus.

Les prélats pointent ainsi le double risque de rabattre le christianisme naissant sur un jeu de pouvoir trop humain et de décrédibiliser l'exégèse biblique auprès des croyants; mais dans le même temps ils considèrent que l'intelligence de la foi appelant la critique historique, les séries d'Arte constituent un outil opportun de questionnement de la tradition et des dogmes. Réserve qui trouve écho dans la communauté universitaire lorsqu'une historienne épingle diverses erreurs ou lorsque, quelques années plus tard, un de ses collègues en vient à dévoiler «l'orientation idéologique »du dernier volet.

Spécialiste reconnue du christianisme antique et de Paul, MarieFrançoise Baslez' rappelle ainsi en 2004 que l'antijudaïsme est d'abord une invention des intellectuels grecs d'Alexandrie dont on retrouve l'écho bien plus tard dans l'antisémitisme chrétien du Moyen Âge mais peu dans les premières communautés chrétiennes où les identités se mêlent. Réduire la naissance du christianisme à un «processus négatif» de ruptures en série (Jésus/sa famille, Hellènes/ Hébreux, Paul/Jacques, etc.) ne rend pas compte d'un mouvement composite d'universalisation du salut dont les sources romaines attestent l'essor bien avant la formalisation des textes canoniques. L'historienne remet ainsi en cause la focalisation du conflit entre Juifs et Grecs sur Jérusalem alors que les tensions autour du message de

9. Voir l'entretien donné à l'hebdomadaire Pèlerin ( $1^{\mathrm{er}}$ avril 2004) puis développé dans Françoise Baslez, "Jésus après Jésus: les révélations d'Arte?", Histoire du christianisme, $\mathrm{n}^{\circ} 21$, avril 2004, p. 6-9. 
Jésus épousent les contours divers des diasporas au contact des cités polythéistes de l'Asie Mineure à Alexandrie en passant par Rome, Antioche, Jérusalem.

Quatre ans après, lors de L'Apocalypse, son collègue de la Sorbonne, Jean-Marie Salamito, va plus loin en contestant ouvertement le schéma qui consiste à dissocier Jésus de la tradition ecclésiale qui se réclame de lui: "Étonnante réalisation, dont les morceaux sont presque tous instructifs et passionnants, mais dont la ligne directrice n'est pas scientifique ${ }^{10}$.» Un vif débat s'ensuit entre le normalien et les réalisateurs, duquel en ressort le premier livre consacré directement à nos séries, les Chevaliers de l'Apocalypse. Réponse à MM. Prieur et Mordillat ${ }^{11}$. L'auteur, qui ne cache pas sa conviction catholique tout en doutant de l'utilité d'une telle précision en la matière, salue d'abord la qualité des intervenants de la série tout en regrettant après bien d'autres leur enrôlement par la voix off sans autre forme de dialogue. Il s'en prend surtout à l'essai des deux «chevaliers " qu'il n'ose pas cependant présenter après d'autres comme les «nouveaux Bouvard et Pécuchet de l'exégèse ».

Leur formule fétiche censée résumer tout («Jésus annonçait le Royaume et c'est l'Église qui est venue ») serait en fait selon lui bâtie sur une contrefaçon de la pensée de Loisy: loin de prôner la rupture entre Jésus et l'Église, le savant exégète inscrivait au contraire cette phrase dans une longue démonstration sur la continuité entre les deux termes par évangiles interposés. Pour Salamito, le refus de voir en face la puissance historique de la foi conduit les «deux compères » à assimiler anachroniquement les martyrs chrétiens à des combattants volontaires et le retrait au désert des premiers moines à une entrée en résistance contre l'Église triomphante des «collaborateurs » de l'Empire. Outre ces anachronismes, l'universitaire récapitule diverses autres erreurs factuelles et de méthode pour condamner finalement la stérilité d'un essai qui se complaît à réduire la naissance du christianisme à une conquête des pleins pouvoirs. Sa leçon d'histoire, agrémentée d'une plume alerte et incisive, ne tarde pas à rencontrer un certain écho: un mois après la sortie du petit livre, Le Monde des livres salue sa pertinence sur presque une demi-page et l'auteur se voit à nouveau invité à débattre avec Mordillat, cette foisci dans l'émission Répliques animée par Alain Finkielkraut sur France Culture ${ }^{12}$.

10. Jean-Marie Salamito, «L’Apocalypse sur écran noir», Le Monde de la Bible, $\mathrm{n}^{\circ}$ 186, 2008, p. 12-16.

11. Notamment lors d'une séance publique au Musée d'art et d'histoire du judaïsme à Paris (6 novembre 2008). Le site internet du Pèlerin Magazine diffuse en fin d'année des extraits vidéo de cette vigoureuse passe d'armes. Le petit livre en question sort en février 2009 chez les éditeurs catholiques Lethielleux et Desclée de Brouwer.

12. Sous le titre «Descente aux enfers pour "L'Apocalypse" de Mordillat et Prieur. L'historien Jean-Marie Salamito dénonce les partis pris et les erreurs d'une série télévisée à succès ", 
Bien avant cet épisode ou épilogue polémique, quelques rares questions s'étaient déjà posées à propos des figures de sens en jeu dans les séries d'Arte - réflexions émanant de philosophes chrétiens engagés dans la revue Esprit. Dès 1997, Paul Thibaud relève ainsi la pauvreté d'un questionnaire qui suppose «qu'il n'y a qu'à vérifier les faits accessibles pour comprendre les récits et les personnages et qu'une expression religieuse est élucidée quand on connaît les quelques faits qui lui servent de support ${ }^{13}$ »; un questionnaire qui ne peut que rendre aveugle à l' «ébranlement existentiel» en jeu dans l'écriture biblique et dont l'écho dépasse les époques et les communautés. En 2004, Jean-Claude Eslin lui emboîte le pas; pour le philosophe, l'explicitation des querelles entre judéo-chrétiens et «paganochrétiens » à travers Paul et les Actes des apôtres, aussi passionnante et utile soit-elle, masque le cœur de l'affaire qui se trouve dans l'écriture plurielle et heurtée des évangiles ${ }^{14}$. Bien que se disant d'abord sensibles à ce que la littérature fait, les écrivains réalisateurs seraient ainsi passés à côté de l'essentiel, l'événement de la loi divine faite homme universel à travers la parole libre puis l'écriture mémorielle et ses interprétations infinies. D'où la réduction de sens que les séries opèrent en rabattant la dialectique du neuf et de l'ancien sur une captation d'héritage.

Certes, les critiques les plus notables de la trilogie peuvent en fin de compte être référées à l'engagement chrétien de leurs auteurs, mais cet état de fait ne saurait atténuer la portée de leurs arguments. Ces derniers qui élèvent l'enquête au niveau de l'enjeu métaphysique de son objet ou à ce que les idées font à l'histoire risquent cependant d'être bien éthérés ou trop complexes au regard du reportage de Paris-Match («Non, Jésus n'était pas antisémite») qui invite nos deux cinéastes transformés pour la circonstance en «historiens agnostiques mais épris de vérité », véritables «puits de science» que la romancière Irène Frain interroge au côté de l'écrivain Marek Halter $^{15}$. C'est sans doute dans ce grand écart entre régimes de vérité et de références (Esprit et Paris-Match) qu'il faut rechercher le public inattendu des séries en question. Et partant un début de réponse à la question faussement naïve de Salamito s'étonnant qu'on puisse

l'historien Maurice Sartre résume et appuie la leçon donnée par son jeune collègue en concluant ainsi : "Dans ces pages rigoureuses et denses, Salamito exerce au mieux son double devoir d'universitaire, celui de chercheur et d'enseignant» (Le Monde des livres, 27 mars 2009). Quant à Répliques (28 mars 2009), grâce sans doute à son animateur qui recentrait le débat sur les tensions originelles entre la communalisation juive de la loi et l'individualisation chrétienne de la foi, la confrontation entre les protagonistes fut moins heurtée que les précédentes, marquant cependant la distance entre l'historien de métier fixé sur les données factuelles d'un mouvement religieux et le cinéaste essayiste sur le dévoilement politique d'une histoire sainte.

13. Paul Thibaud, «Jésus chez les modernes ", Esprit, mai 1997, p. 187-188.

14. Jean-Claude Eslin, "L'origine du christianisme: réflexions à partir d'une enquête de Gérard Mordillat et Jérôme Prieur », Esprit, juillet 2004, p. 29-43.

15. Paris-Match, «Le Christ sans passion: non, Jésus n'était pas antisémite », 22-28 avril 2004 , p. 27-30. 
«mobiliser tant de moyens, tant de savants, tant d'heures de tournage, tant d'heures d'antenne, tant d'images superbes, tant de pages de livres, tant de publicité, pour asséner à tous, en fin de compte, sur le christianisme antique, des conclusions dignes du café du Commerce!» (p. 154).

Après le succès de Corpus Christi et son million et plus de téléspectateurs estimés par Médiamat le public s'est fidélisé puis tassé en fin de parcours ${ }^{16}$. À titre indicatif et non comparatif, notons que si la première série "surprise " a dépassé les prévisions de la chaîne (5\% de part de marché réalisée en moyenne contre $3 \%$ prévue) une fiction réaliste comme L'instit sur France 2 réalisait par exemple à la même heure en 1997 un niveau d'audience dix fois plus élevé. Si pour les sondeurs le public de nos séries est «ciblé » (plus de 50 ans, professions intellectuelles, grandes villes) et fortement incité par une campagne de presse hors pair, ses caractères propres ne sont pas sans lien avec les prises de position qui ont cours lors de la réception ${ }^{17}$. Cohabitent ainsi les cercles d'admiration qui privilégient le kaléidoscope des paroles savantes comme éthique du doute et spectacle de l'intelligence, les bataillons de la tradition qui se justifient dans l'exécration de la pensée critique, les militants de la raison laïque qui se réjouissent que se lève enfin au grand jour un dernier coin de voile sur une légende trop longtemps entretenue, les initiés qui dévoilent en retour le stratagème médiatique qui se joue de leur connaissance en même temps que de la foi religieuse. Sans doute la notoriété de nos séries doit-elle autant à l'opposition plus ou moins polémique de ces motifs qu'à leur entremêlement discret comme le montrent les humeurs enjouées de certains blogueurs en ligne ${ }^{18}$.

Au cœur de cet entre-deux ne s'affirme pas moins une soif de savoir qui habite une partie de la vieille Europe sécularisée, notamment en France où l'antagonisme entre l'Église et l'État a perturbé la connaissance objective des sources chrétiennes. Si tant est que la «sainte ignorance", comme le dit Olivier Roy, ait pris pied dans un espace culturel vidé de ses anciennes querelles entre la foi et la raison, l'événement d'Arte ne révèle pas moins un des résidus actifs de

16. Avec toutes les réserves qu'implique ce type d'estimation, Corpus Christi et L'origine du christianisme ont capté en moyenne 1 à 1,5 million de téléspectateurs et 3 à $5 \%$ de part de marché. La constance de l'audience entre les deux séries est remarquable. L'Apocalypse n'a capté que 0,5 million et $2,1 \%$ de part de marché, baisse qui s'explique en grande partie par le redéploiement télévisuel, entre-temps, en d'innombrables canaux numériques.

17. Nous rejoignons en cela tout un courant de la sociologie des publics qui fonde une part de ses données sur les phénomènes de réception (voir Jean-Pierre Esquenazi, Sociologie des publics, Paris, La Découverte, coll. "Repères », 2003).

18. On peut par exemple citer "Sex and the Cristi" ou "Constantin se convertit aux délocalisations », présentations hilarantes de Samuel Gontier sur Télérama.fr qui se jouent avec talent des anachronismes dénoncés par les universitaires. Autre genre, «Jésus, les égorgeurs et l'art de la citation " (philosophie.blogs.liberation) où le linguiste Pierre Encrevé donne une leçon de citation à propos d'une interview de nos deux réalisateurs et déclenche une suite d'appréciations contrastées sur les séries en question. 
cet espace, celui qui fait l'auditoire aussi croissant que grisonnant des cours d'histoire des traditions bibliques à l'Institut catholique, à la Sorbonne ou au Collège de France ${ }^{19}$. «Cœur de cible» des séries artésiennes dans lequel se logent ses plus chauds partisans, mais aussi ses premiers déçus, ne serait-ce que pour marquer leur distance avec une excellente œuvre de vulgarisation qui au fil du temps s'est avérée, aux dires de certains de leurs professeurs, par trop partiale.

\section{Fin de parcours?}

L'image d'une comète résume assez bien notre affaire: petit astre brillant accompagné d'une traîne lumineuse et dont l'orbite a la forme d'une ellipse. Le brillant c'est pour la qualité de la mise en scène du savoir et de ses acteurs sollicités; la traîne c'est pour l'écho public rencontré par les séries ; l'ellipse c'est pour le parcours qui va du succès d'audience jusqu'à son relatif déclin, sur fond de polémique savante. Après Corpus Christi, les réalisateurs hésitaient à répondre à la question de savoir s'ils donneraient une suite à leur surprenante initiative; après L'Apocalypse, ils estiment leur mission accomplie au terme d'une trilogie qui les a investis pendant une bonne quinzaine d'années. On peut se demander si, outre le sentiment d'avoir fait le tour d'une question, le tassement relatif de l'audience et la levée de boucliers finale de quelques universitaires ne les ont pas rendu plus affirmatifs. Ils n'en ressortent pas moins auréolés d'un titre national d'experts en christianisme primitif décerné par les grands prescripteurs d'opinion, qualité rare et que peu d'universitaires ont acquis depuis Renan ${ }^{20}$. Mieux encore, le Centre national de documentation pédagogique prend la série comme base de cours d'histoire et de lettres dans les lycées ${ }^{21}$.

En ces temps troublés où l'idée de «laïcité positive» est avancée par la potestas d'État pour s'attirer les bonnes grâces de l'auctoritas morale de la principale Église, nos deux experts usent de leur notoriété acquise pour tourner en dérision les vestiges de dévotion. Lors d'une désopilante conférence publique dans la salle Topor du théâtre du Rond-Point (juin 2008), l'un répond à l'autre, "professeur du cercle Voltaire de l'École biblique hors-les-murs » grattant sa barbe postiche, pour savoir si la crucifixion de Jésus peut être vraiment

19. Olivier Roy, la Sainte ignorance. Le temps de la religion sans culture, Paris, Le Seuil, coll. « La couleur des idées », 2008.

20. Les réalisateurs ont ainsi été invités par diverses institutions prestigieuses comme la villa Médicis à Rome, la Bibliothèque nationale de France, le musée du Louvre, la fondation Bodmer, le centre Rachi, les universités de Tübingen, Genève, Lausanne, Jérusalem, etc.

21. Dossier L'Apocalypse mis en ligne sur Télédoc 2008-2009 par le Centre national de documentation pédagogique. 
considérée comme un accident du travail ${ }^{22}$. Les «Rivoire et Carret de l'exégèse » confirment ainsi leur rôle iconoclaste en participant à l'opération nationale «Rire de résistance» lancé par Jean-Michel Ribes directeur du théâtre parisien ${ }^{23}$. Faut-il y voir un lien avec le fait que ce soit l'Association des amis du journal L'Humanité plutôt que l'Institut catholique de Paris qui ait invité nos réalisateurs à présenter en avant-première leur troisième et dernier volet ${ }^{24}$ ? Ce serait sans doute trop simple (les facultés catholiques de province ne les ont pas moins invités) et il serait évidemment bien triste de terminer ce petit parcours sur un vague et désuet écho de la «lutte des deux France». Dans l'attente du temps qui manque pour appréhender l'empreinte que les séries d'Arte laisseront dans la mémoire collective, quelques conclusions provisoires s'imposent cependant.

Longtemps approprié par les Églises, le savoir sur les origines du christianisme a naturellement suivi la voie de la sécularisation en se renouvelant avec le mouvement européen de Critica sacra lancé à la Renaissance et systématisé plus tard par Spinoza ${ }^{25}$. Depuis le XIX ${ }^{\mathrm{e}}$ siècle, il s'est très partiellement déplacé des institutions confessionnelles vers les marges de l'Université (création entre autres en 1886 d'une section de «sciences religieuses" dans la très républicaine École pratique des hautes études). La séparation des Églises et de l'État a en effet paradoxalement freiné en France le transfert de savoir critique (au sens positif du terme) vers les institutions laïques d'enseignement et ce pour des raisons indissociablement politiques (apaiser les conflits passionnels autour de la principale religion du pays) et techniques (offre insuffisante de compétences par rapport à la demande). Cet héritage conflictuel entrave encore aujourd'hui les dispositifs de connaissance historique et de sa transmission publique au moment où le christianisme n'a pourtant plus beaucoup d'autorité morale mais où se ravivent aussi les passions religieuses dans un pays enrichi de flux migratoires multiples.

On ne peut donc s'étonner qu'il soit revenu à nos deux réalisateurs d'ouvrir une fenêtre sur l'immense savoir biblique accumulé à l'ombre des facultés de théologie et de quelques départements d'histoire

22. Un autre petit livre en laisse une trace: Jérôme Prieur, Gérard Mordillat, De la crucifixion considérée comme un accident du travail, Paris, Démopolis, 2008.

23. Erwan Desplanques et Michel Abescat, «Quand on n'a que l'humour. D'Aristophane à Coluche, on a toujours raillé le pouvoir. Pour tenir. Même face aux pires tyrannies. Le point sur ce rire de résistance, à l'occasion des conférences azimutées du théâtre du Rond-Point », Télérama, 3017, 2007, p. 24-30.

24. Le 13 décembre 2008, à Paris et au cinéma des 3 Luxembourg.

25. Sur l'histoire de la critique biblique: François Laplanche, la Bible en France. Entre mythe et critique, $X V T^{e}-X I X^{e}$ siècles, Paris, Albin Michel, coll. "L'évolution de l'humanité », 1994; la Crise de l'origine. La science catholique des Évangiles et l'histoire au XX $X^{e}$ siècle, Paris, Albin Michel, coll. "L'évolution de l'humanité », 2006. Sur sa sociologie: Pierre Lassave, "Ce que les Écritures saintes font à leur science. Vers une sociologie de l'exégèse biblique ", Archives de sciences sociales des religions, $\mathrm{n}^{\circ} 139,2007$, p. 47-66. 
antique de par le monde. Athées mais fascinés par l'énigme de Jésus, autodidactes en exégèse biblique mais férus de littérature, cinéastes mais aussi écrivains, ces passeurs de frontières ont bien le profil ambivalent de ceux qui dénouent l'intrigue en même temps qu'ils la relancent: tricksters ou «décepteurs» pour l'anthropologue, «embrayeurs paratopiques» pour le théoricien du récit, «connecteurs opportunistes de réseaux » pour le sociologue ${ }^{26}$. L'ouverture qu'ils ont opérée ne devrait pas à terme rester sans effet sur les consciences et les institutions.

Mais l'opposition des termes entre le Royaume annoncé par Jésus et l'Église qui a pris sa place, fil rouge de la trilogie difficilement contestable dans une acception positiviste de l'histoire, limite quelque peu l'horizon au vieux débat entre les tenants du Jésus historique et ceux du Christ de la foi. Les nombreux savants qu'ils ont sollicités n'ont-ils pas montré tant par leur recherche à vie, leurs doutes éternels et leurs analyses intensives de témoins textuels que le Christ de la foi est la principale énigme historique à résoudre bien au-delà de la nécessaire déconstruction des traditions et de leurs raisons politiques? Des sources historiques qui relèvent plus que d'autres d'actes de foi n'exigent-elles pas d'être mises en tant que telles au centre de l'enquête plutôt que de s'épuiser ou de s'en tenir à déterminer ce qu'elles masquent? Sans doute qu'en la matière l'anthropologie est bien trop en retrait de l'histoire et de la théologie sur la scène universitaire pour relever un tel défi de connaissance ${ }^{27}$.

Durkheim avait pourtant lui aussi ouvert une fenêtre en montrant tout ce que nos catégories de raisonnement (séparation, analogie, classement) doivent aux formes soi-disant «élémentaires » de la vie religieuse. Formes «supplémentaires », les sources canoniques, apocryphes ou apologétiques ("écriture, dangereux supplément de la parole» écrivait Rousseau) ne s'offrent-elles pas encore à la découverte de ce que leurs multiples Jésus et Christ ont fait à notre propre histoire, y compris la plus savante? Sur ce chemin, les comètes médiatiques et leurs traînes de presse s'avèrent éclairantes, ne seraitce que pour montrer une distance à parcourir.

Pierre Lassave

26. Voir Paul Radin, The Trickster: A Study in American Indian Mythology with Commentaries by K. Kerenyi and C.G. Jung, Londres, Routledge et Paul Kegan, 1956 (trad. fr. : le Fripon divin, texte complet du mythe des Indiens Winnebago, Genève, Georg, 1958); Dominique Maingueneau, le Contexte de l'œuvre littéraire. Énonciation, écrivain, société, Paris, Dunod, 1993; Luc Boltanski et Ève Chiapello, le Nouvel esprit du capitalisme, Paris, Gallimard, coll. « NRFEssais ", 1999.

27. Camille Tarot, De Durkheim à Mauss, l'invention du symbolique, Paris, La Découverte, coll. «Recherches », 1999. 


\section{Une analyse polémique*}

D'entrée de jeu, il faut signaler que Jean-Marie Salamito critique certes et attaque, en historien, les émissions télévisées intitulées L'Apocalypse, mais surtout le livre correspondant de Gérard Mordillat et Jérôme Prieur, Jésus sans Jésus ${ }^{1}$. Concernant les émissions, il fait des réserves, comme beaucoup d'autres, sur la confection artificielle, pour la télévision, d'un dispositif qui consiste à placer les savants dans une sorte de chambre noire, sans qu'il y ait jamais débat entre eux. Cette façon de mettre en condition l'intervenant peut mettre mal à l'aise et celui-ci et le téléspectateur.

Le paradoxe des émissions de Mordillat et Prieur est qu'une grande pluralité d'interprétations est donnée par la quarantaine de spécialistes interrogés, qu'ainsi pour la première fois, le grand public a accès à cette pluralité, d'où une impression d'ouverture, d'esprit de recherche, de complexité; mais qu'en même temps on éprouve que l'ensemble des émissions est soutenu par une interprétation personnelle des auteurs, hostile au christianisme, qui est la leur, non celle des intervenants, et ne s'impose pas.

Le livre de J.-M. Salamito sera précieux pour qui a vu les émissions télévisées. Il est une sorte de commentaire critique qui, page après page, complexifie et corrige ce qui est dit. La critique s'articule à juste titre sur le point majeur. Mordillat et Prieur font dire à la phrase de Loisy qui leur sert de fétiche: «Jésus annonçait le royaume, et c'est l'Église qui est venue», tirée de son contexte, exactement le contraire de ce qu'elle dit. Salamito cite la page entière de l'Évangile et l'Église (1902) et tout est vu dans une autre lumière :

Reprocher à l'Église catholique tout le développement de sa constitution, c'est lui reprocher d'avoir vécu, ce qui pourtant ne laisse pas d'être indispensable à l'Évangile même. [...] Jésus annonçait le royaume, et c'est l'Église qui est venue. Elle est venue en élargissant la forme de l'Évangile qu'il était impossible de garder telle quelle, dès que le ministère de Jésus eut été clos par la passion. Il n'est aucune institution sur la terre, ni dans l'histoire des hommes dont on ne puisse contester la légitimité et la valeur, si l'on pose en principe que rien n'a droit d'être vu que dans son état originel.

Contrairement à Mordillat et Prieur, Loisy, en 1902, légitime le développement et reconnaît la continuité entre l'Évangile et l'Église.

Pour l'exactitude, il faut reconnaître que parfois les rectifications de Jean-Marie Salamito sont incomplètes. Il défend à juste titre l'attitude d'Ambroise, évêque de Milan, devant l'empereur dans l'affaire du massacre de Thessalonique. Mais il ne mentionne pas l'affaire de la synagogue de Calliacum qui témoigne de la part de l'évêque d'une volonté d'ouvrir un front d'antijudaïsme: Ambroise fait pression sur l'empereur pour éviter la réparation d'une synagogue incendiée par des chrétiens.

Autre détail, la déclaration de la faculté de théologie catholique de Lyon en juin 1941 au moment du second statut des juifs n'a pas été à proprement parler publiée : cela a paru impossible. Elle a été diffusée, assez discrètement $^{2}$.

* À propos de Jean-Marie Salamito, les Chevaliers de l'Apocalpyse. Réponse à MM. Prieur et Mordillat, Paris, Lethielleux/Desclée de Brouwer, 2009, 160 p., $12 €$.

1. G. Mordillat et J. Prieur, Jésus sans Jésus. La christianisation de l'Empire romain, Paris, Le Seuil-Arte éditions, 2008.

2. Voir H. de Lubac, Résistance chrétienne à l'antisémitisme, Paris, Fayard, 1988. 
Jésus après Arte. Retour sur une trilogie

Après les polémiques et les oppositions frontales comme à l'émission «Répliques» le 28 mars, je me demande si la première histoire chrétienne peut être lue de façon neutre. Deux visions s'opposent : ou bien on y voit, au milieu de circonstances contingentes, l'inscription sur terre d'une réalité christique qui laboure profondément et spirituellement le sol antique; ou bien ou y voit comme déjà Gibbon une sorte de catastrophe pour l'Empire romain et le judaïsme, une défaite de l'esprit de tolérance. Deux visions incompatibles: qu'on le veuille ou non, l'affaire Jésus met toujours à l'épreuve.

Jean-Claude Eslin 